\title{
LOW ENERGY EXCITATION SPECTRA OF TRAPPED BOSE CONDENSATES
}

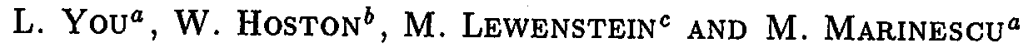 \\ ${ }^{a}$ School of Physics, Georgia Institute of Technology, Atlanta, GA 30332-0430, USA \\ ${ }^{b}$ Institute for Theoretical Atomic and Molecular Physics \\ Harvard-Smithsonian Center for Astrophysics \\ 60 Garden Street, MS 14, Cambridge, Massachusetts 02138, USA \\ ${ }^{\circ}$ Commissariat à l'Energie Atomique, DSM/DRECAM/SPAM \\ Centre d'Etudes de Saclay \\ 91191 Gif-sur-Yvette, France
}

\begin{abstract}
We discuss our numerical studies of the low energy excitations of trapped Bose condensates using a Bogoliubov-Hartree treatment. In the zero temperature limit, the lowest few excitation frequencies calculated within the Bogoliubov approximation agree well with the experimental data. Finite temperature results obtained using the Popov approximation display qualitative differences from the experimental data close to the critical temperature region. Details of our numerical approach are presented and comparison with other results is discussed.
\end{abstract}

PACS numbers: 03.75.Fi, 67.90.+z

\section{Introduction}

A little over two years ago a new phase of research was entered by experimentalists pursuing research in the Bose-Einstein condensation (BEC). Built upon cumulative research advances in laser cooling and trapping [1] and in evaporative cooling [2], BEC was first observed in ${ }^{87} \mathrm{Rb}$ by a collaboration at JILA [3]. Shortly thereafter, a group at Rice University reported apparent evidence of quantum degeneracy with ${ }^{7} \mathrm{Li}[4]$, and a group at MIT developed techniques for rapid production of large condensates containing about a million ${ }^{23} \mathrm{Na}$ atoms [5]. Tremendous progress has been made in the past two years: many single particle properties of trapped condensates have been measured; direct non-destructive optical imaging techniques have been developed [6]; several of the low-lying collective excitation modes have been detected $[7,8]$. Recently, the macroscopic coherence properties of the condensate were displayed in a spectacular fashion with the demonstration of interference between two condensates [9], and the suppression of the collisional losses from the inelastic collisions [10] due to the multi-particle correlations. Rudimentary atom lasers with pulsed output couplers have also been reported $[9,11]$, and the sound velocities of the condensate have been measured [12]. 
The theoretical description of these experimental systems has also been an active field. Particular interest has been focused on the calculation of excitation spectra for condensates. In the zero temperature limit, the Bogoliubov-de Gennes equations that govern the wave functions for quasi-particles have been studied by several groups $[13,14]$. An (analytic) asymptotic expression for the eigenfrequencies valid in the Thomas-Fermi/hydrodynamic limit was first presented by Stringari [15]. A variational approach was developed in Ref. [16]. A dynamical variational approach was also applied by Pérez-García et al. [17]. More recent studies can be found in Refs. [18-21]. All of these studies describe the condensate within a mean-field approximation with excitations corresponding to the poles of the single particle Green function. Multi-particle excitations and related correlation effects have not been carefully addressed yet, although an atomic structure approach based on the Hartree-Fock, random phase approximation, and configuration interaction approach as presented in [22], can be used to study such correlation effects by including multi-excitation configurations [23]. The finite temperature mean-field Bogoliubov-Hartree (BH) approach to the collective excitations involves a great deal of numerical effort and only limited results are available at the moment [24]. A detailed comparison with the experimental data [8] is yet to be performed.

In this paper we present the numerical procedure we have developed for the study of low energy excitations of trapped Bose condensates [25]. The paper is organized as follows. We start by giving an overview of the field theoretical description of the condensate. In Sec. 3 we follow with an outline of the numerical procedures we have developed. The results and discussions are given in Sec. 4. Finally, we conclude in Sec. 5 .

\section{Bogoliubov-Hartree theory}

Several versions of the Bogoliubov-Hartree theory exist in the literature [26] but we closely follow the approach of Ref. [25], which is based on the linearization of the 2nd-quantized Hamiltonian around a coherent state (or a $c$-number) of the atomic fields. This approach fixes the overall phase (by breaking the global U(1) gauge invariance) of the mean atomic fields and keeps quadratic terms of the small quantum fluctuations (around this coherent state) in the Hamiltonian. For trapped systems, these quantum fluctuations cause the initial fixed phase of the atom field to diffuse, which can be understood mathematically as due to the degeneracy of the zero mode in the quasi-particle excitation spectra. For a detailed discussion of the zero mode, see Ref. [27]. Recently approaches which conserve the number of particles (and therefore do not fix the phase) have been presented [28].

The second quantized Hamiltonian for a system of $N$ spinless bosonic atoms trapped in a potential $V_{t}(r)$ is given by $[25,26]$

$$
\begin{aligned}
\mathcal{H}= & \int \mathrm{d} \boldsymbol{r} \widehat{\Psi}^{\dagger}(\boldsymbol{r})\left[-\frac{\hbar^{2}}{2 M} \nabla^{2}+V_{t}(\boldsymbol{r})-\mu\right] \widehat{\Psi}(\boldsymbol{r}) \\
& +\frac{u_{0}}{2} \int \mathrm{d} \boldsymbol{r} \widehat{\Psi}^{\dagger}(\boldsymbol{r}) \hat{\Psi}^{\dagger}(\boldsymbol{r}) \widehat{\Psi}(\boldsymbol{r}) \widehat{\Psi}(\boldsymbol{r}),
\end{aligned}
$$


where $\widehat{\Psi}(\boldsymbol{r})$ and $\widehat{\Psi}^{\dagger}(\boldsymbol{r})$ are atomic (bosonic) annihilation and creation fields, $u_{0}=$ $4 \pi \hbar^{2} a_{\mathrm{sc}} / M$, with $M$ being the atomic mass, and $a_{\mathrm{sc}}$ - the scattering length of the atom-atom interaction. This Hamiltonian in fact describes the free energy since the chemical potential $\mu$ is used to guarantee the conservation of the average of the total number of atoms $\widehat{N}=\int \mathrm{d} r \widehat{\Psi}^{\dagger}(r) \widehat{\Psi}(r)$. The aim of the BH approach is to describe the single particle excitations of the system in terms of non-interacting quasiparticles. Mathematically we try to cast the Hamiltonian (2.1) into the form

$$
\mathcal{H} \rightarrow \sum_{n \neq 0} \hbar \tilde{\omega}_{n} \tilde{g}_{n}^{\dagger} \tilde{g}_{n}+\left(n=0 \text { zero mode part } \alpha \widehat{P}^{2} / 2\right),
$$

where $\tilde{g}_{n}^{\dagger}\left(\widetilde{g}_{n}\right)$ are the quasiparticle creation (annihilation) operators which satisfy the standard bosonic commutation relations and the quasiparticle index, $n=0,1,2, \ldots$, labels the positive eigenfrequencies $\widetilde{\omega}_{n}$ arranged in ascending order. Therefore, the density matrix for quasiparticles at equilibrium is described by the Bose-Einstein distribution

$$
\widehat{\rho}=\frac{1}{Z} \exp \left(-\beta \sum_{n \neq 0} \hbar \widetilde{\omega}_{n} \tilde{g}_{n}^{\dagger} \widetilde{g}_{n}+\ldots\right),
$$

where $\widetilde{\omega}_{n}$ depend on the chemical potential $\mu$ and $Z$ is the partition function.

At zero temperature the $\mathrm{BH}$ approach takes its simplest form, the Bogoliubov approximation, which starts with the assumption

$$
\widehat{\Psi}(r)=\sqrt{N_{0}} \psi_{0}(r)+\delta \widehat{\Psi}(r),
$$

where the $c$-number condensate wave function $\psi_{0}(r)$ is assumed to be real (without loss of generality) and normalized such that $\int \mathrm{d} r\left|\psi_{0}(r)\right|^{2}=1$, and $N_{0}$ is the number of particles in the condensate. In Eq. $(2.4), \delta \widehat{\Psi}(r)$ denotes the quantum fluctuation which obeys the same standard bosonic commutation relations as $\widehat{\Psi}(r)$. We substitute Eq. (2.4) into Eq. (2.1) and neglect both 3-rd and 4-th order fluctuation terms. The linear fluctuation terms are vanishing provided that $\psi_{0}(r)$ satisfies the nonlinear Schrödinger equation (NLSE), i.e.

$$
\left[\mathcal{L}+u_{0} \rho_{0}\right] \psi_{0}(r)=0
$$

where we have defined $\mathcal{L} \equiv-\frac{\hbar^{2} \nabla^{2}}{2 M}+V_{t}(r)-\mu$, and the condensate density $\rho_{0}(r) \equiv$ $N_{0}\left|\psi_{0}(r)\right|^{2}$. The resulting linearized effective Hamiltonian

$$
\begin{aligned}
\mathcal{H}_{\mathrm{eff}} & =\int \mathrm{d} r \delta \widehat{\Psi}^{\dagger}(r) \mathcal{L} \delta \widehat{\Psi}(r)+\frac{1}{2} N u_{0} \int \mathrm{d} r \psi_{0}^{2}(r) \\
& \times\left[\delta \hat{\Psi}^{\dagger}(r) \delta \widehat{\Psi}^{\dagger}(r)+\text { h.c. }+4 \delta \widehat{\Psi}^{\dagger}(r) \delta \widehat{\Psi}(r)\right],
\end{aligned}
$$

may be diagonalized in the representation of the quasiparticle annihilation operators

$$
\widetilde{g}_{k}=\int \mathrm{d} \boldsymbol{r}\left[U_{k}(r) \delta \widehat{\Psi}(r)+V_{k}(r) \delta \widehat{\Psi}^{\dagger}(r)\right],
$$

for $k=1,2,3, \ldots$ and their Hermitian conjugates, a quasiparticle creation operators $\widetilde{g}_{k}^{\dagger}$. The $U_{k}(r)$ and $V_{k}(r)$ are the mode functions of the quasiparticles which 
have to be determined. To determine the functions $U_{n}(r)$ and $V_{n}(r)$ (for $n \neq 0$ ) we solve $\left[\tilde{g}_{n}, \mathcal{H}\right]=\hbar \tilde{\omega}_{n} \tilde{g}_{n}$, which is equivalent to the coupled Bogoliubov-de Gennes equations

$$
\begin{aligned}
& {\left[\mathcal{L}+2 u_{0} \rho_{0}(\boldsymbol{r})\right] U_{n}(\boldsymbol{r})-u_{0} \Delta_{0}^{*}(\boldsymbol{r}) V_{n}(\boldsymbol{r})=\hbar \widetilde{\omega}_{n} U_{n}(\boldsymbol{r}),} \\
& {\left[\mathcal{L}+2 u_{0} \rho_{0}(\boldsymbol{r})\right] V_{n}(\boldsymbol{r})-u_{0} \Delta_{0}(\boldsymbol{r}) U_{n}(\boldsymbol{r})=-\hbar \tilde{\omega}_{n} V_{n}(\boldsymbol{r}),}
\end{aligned}
$$

where $\Delta_{0}(r)=N_{0} \psi_{0}^{2}(r)$. There is a time-reversal symmetry associated with (2.8). If the set $\left\{U_{n}(r), V_{n}(r)\right\}$ constitutes a solution for energy $+\hbar \widetilde{\omega}_{n}$ then the set $\left\{V_{n}^{*}(r), U_{n}^{*}(r)\right\}$ is also a solution but for energy $-\hbar \tilde{\omega}_{n}$ [26]. All of the non-zero eigenvalues are thus paired and real. To have $\widetilde{g}_{k}$ and $\tilde{g}_{k}^{\dagger}$ fulfill bosonic commutation relations, $\left[\widetilde{g}_{k}, \widetilde{g}_{k^{\prime}}^{\dagger}\right]=\delta_{k k^{\prime}},\left[\widetilde{g}_{k}, \widetilde{g}_{k^{\prime}}\right]=0$, the mode functions have to obey the orthonormality condition [26],

$$
\begin{aligned}
& \int \mathrm{d} \boldsymbol{r}\left[U_{k}(\boldsymbol{r}) U_{k^{\prime}}^{*}(\boldsymbol{r})-V_{k}(\boldsymbol{r}) V_{k^{\prime}}^{*}(\boldsymbol{r})\right]=\delta_{k k^{\prime}}, \\
& \int \mathrm{d} \boldsymbol{r}\left[U_{k}(\boldsymbol{r}) V_{k^{\prime}}(\boldsymbol{r})-V_{k}(\boldsymbol{r}) U_{k^{\prime}}(\boldsymbol{r})\right]=0 .
\end{aligned}
$$

The presence of a zero mode solution to Eq. (2.8) requires the introduction of the momentum operator $\widehat{P}$, defined according to [27] as,

$$
\widehat{P}=\int \mathrm{d} \boldsymbol{r} \psi_{0}(r)\left[\delta \widehat{\Psi}(r)+\delta \widehat{\Psi}^{\dagger}(r)\right] .
$$

The zero mode and the above associated momentum operator describe collective motion without restoring force of the condensate [29]. Therefore, $\widehat{P}$ commutes with all $\widetilde{g}_{k}$, and $\widetilde{g}_{k}^{\dagger}$ operators, i.e., $\int \mathrm{d} \boldsymbol{r} \psi_{0}(\boldsymbol{r})\left[U_{k}(\boldsymbol{r})-V_{k}(\boldsymbol{r})\right]=0$ for $k \neq 0$. The conjugate "position" operator is defined as

$$
\widehat{Q}=\mathrm{i} \int \mathrm{d} \boldsymbol{r} \Phi_{0}(r)\left[\delta \widehat{\Psi}(r)-\delta \widehat{\Psi}^{\dagger}(r)\right],
$$

and it has to satisfy $[\widehat{Q}, \widehat{P}]=\mathrm{i},\left[Q, \widetilde{g}_{k}\right]=0($ for $k \neq 0)$, and $\left[\widehat{Q}, \mathcal{H}_{\mathrm{eff}}\right]=\mathrm{i} \alpha \widehat{P}[27]$. Thus, we obtain

$$
\begin{aligned}
& 2 \int \mathrm{d} r \Phi_{0}(r) \psi_{0}(r)=1 \\
& \int \mathrm{d} r \Phi_{0}(r)\left[U_{k}(r)+V_{k}(r)\right]=0, \quad \text { for } k \neq 0,
\end{aligned}
$$

and

$$
\left[\mathcal{L}+3 u_{0} \rho_{0}(r)\right] \Phi_{0}(r)=\alpha \psi_{0}(r)
$$

which has a unique solution, since the operator on the lhs of Eq. (2.13) is clearly positive-definite. The coefficient $\alpha$ is related to the rate of change of the condensate phase. The annihilation operator for the zero mode is $\widetilde{g}_{0}=(\widehat{P}-\mathrm{i} \widehat{Q}) / \sqrt{2}$. Its associated mode functions are given by

$$
\begin{aligned}
& U_{0}(r)=\left[\psi_{0}(r)+\Phi_{0}(r)\right] / \sqrt{2} \\
& V_{0}(r)=\left[\psi_{0}(r)-\Phi_{0}(r)\right] / \sqrt{2}
\end{aligned}
$$


The total atomic field can now be expanded as

$$
\widehat{\Psi}(\boldsymbol{r})=\sum_{k=0}^{\infty}\left[U_{k}(\boldsymbol{r}) \widetilde{g}_{k}-V_{k}(\boldsymbol{r}) \tilde{g}_{k}^{\dagger}\right],
$$

assuming the validity of the linearization approximation. Since the mean value of $\widehat{\Psi}(\boldsymbol{r})$ is $\sqrt{N_{0}} \psi_{0}(\boldsymbol{r})$, this can happen if and only if the system is in the coherent state $\left|\sqrt{N_{0}}\right\rangle$ of all the quasiparticle operators $\widetilde{g}_{k}\left|\sqrt{N_{0}}\right\rangle=z_{k}\left|\sqrt{N_{0}}\right\rangle$, such that

$$
\sum_{k=0}^{\infty}\left[z_{k} U_{k}(\boldsymbol{r})-z_{k}^{*} V_{k}(\boldsymbol{r})\right]=\sqrt{N_{0}} \psi_{0}(\boldsymbol{r})
$$

The above condition implies that $z_{k}=\sqrt{N_{0}} \int \mathrm{d} r \psi_{0}(\boldsymbol{r})\left[U_{k}(\boldsymbol{r})+V_{k}(\boldsymbol{r})\right]$ and $z_{k}^{*}=z_{k}$.

In addition to the collective excitation frequencies $\widetilde{\omega}_{n}$, several other quantities are of potential interest. The total energy of the system (at $T=0$, the ground state) of trapped atoms is given by

$$
\mathcal{E}_{0}=N_{0} \mu+\mathcal{H}_{0}=N_{0} \mu-u_{0} \int \mathrm{d} \boldsymbol{r} \rho_{0}^{2}(\boldsymbol{r}) / 2,
$$

which may be measured as the total release energy by turning off the trapping potential [30]. The depletion of the condensate (caused by the inter-atomic interactions) at zero temperature is [31],

$$
N^{\prime}(T=0)=\int \mathrm{d} r \sum_{n>0}\left|V_{n}(r)\right|^{2} .
$$

For $T \neq 0$, a self-consistent Bogoliubov-Hartree approach is usually adopted. Within the present notation, we have to take into account the neglected 3rd and 4th order terms of $\delta \Psi(r)$ and $\delta \Psi^{\dagger}(r)$ and reduce these terms to quadratic operator terms using a decorrelation approximation [26]. The resulting equations will then be slightly different from those used for the zero temperature studies. First the condensate wave function $\sqrt{N_{0}} \psi_{0}(r)$ cannot in general be assumed to be real. In fact, the analogue to the NLSE are two coupled equations

$$
\begin{aligned}
& {\left[\mathcal{L}+u_{0}\left(\rho_{0}+2 \rho^{\prime}\right)\right] \psi_{0}(r)+u_{0} \Delta^{\prime}(r) \psi_{0}^{*}(r)=0,} \\
& {\left[\mathcal{L}+u_{0}\left(\rho_{0}+2 \rho^{\prime}\right)\right] \psi_{0}^{*}(r)+u_{0} \Delta^{\prime *}(r) \psi_{0}(r)=0 .}
\end{aligned}
$$

Similarly modifications to Eqs. (28) lead to

$$
\begin{aligned}
& {\left[\mathcal{L}+2 u_{0} \rho(\boldsymbol{r})\right] U_{n}(\boldsymbol{r})-u_{0} \Delta^{*}(\boldsymbol{r}) V_{n}(\boldsymbol{r})=\hbar \widetilde{\omega}_{n} U_{n}(\boldsymbol{r}),} \\
& {\left[\mathcal{L}+2 u_{0} \rho(\boldsymbol{r})\right] V_{n}(\boldsymbol{r})-u_{0} \Delta(\boldsymbol{r}) U_{n}(\boldsymbol{r})=-\hbar \widetilde{\omega}_{n} V_{n}(\boldsymbol{r}),}
\end{aligned}
$$

where we have used the notations

$$
\begin{aligned}
& \rho(r)=\rho_{0}(r)+\rho^{\prime}(r), \quad \rho^{\prime}(r)=\sum_{n \geq 0}\left[N_{n}\left|U_{n}(r)\right|^{2}+\left(N_{n}+1\right)\left|V_{n}(r)\right|^{2}\right], \\
& \Delta(r)=\Delta_{0}(r)+\Delta^{\prime}(r), \quad \Delta^{\prime}(r)=-\sum_{n \geq 0}\left(2 N_{n}+1\right) U_{n}^{*}(r) V_{n}(r),
\end{aligned}
$$

where

$$
N_{n \neq 0}=\left\langle g_{n}^{\dagger} g_{n}\right\rangle=\frac{1}{\mathrm{e}^{\beta \hbar \tilde{\omega}_{n}}-1}, \quad \beta=1 / k T,
$$


is the Bose-Einstein distribution. We also obtain

$$
\begin{aligned}
& \mathcal{E}_{0}=N_{0} \mu-u_{0} \int \mathrm{d} r \rho_{0}(r)\left[\rho_{0}(r) / 2+\Delta^{\prime}(r)+2 \rho^{\prime}(r)\right], \\
& N^{\prime}(T)=\int \mathrm{d} r \rho^{\prime}(r) .
\end{aligned}
$$

Self-consistent solutions to the above Eqs. (2.19) and (2.20) can be obtained through an iterative scheme. We note that the $\mathrm{BH}$ approximation as outlined here is a conserving approximation, but it leads to an energy gap in the excitation spectra [26]. Generally, one expects the low energy excitations for an interacting Bose gas to be gapless since the zero mode will always be present in a $\mathrm{U}(1)$ symmetry-breaking approach $[26,27]$. A commonly adopted approximation to enforce gapless excitation solutions is by setting $\Delta^{\prime}=0$ in Eqs. (2.19) and (2.20). Such an approach is called the Popov approximation. It is gapless but this is not a conserving approximation. However, it has allowed preliminary numerical studies of finite temperature excitation spectra [24]. Recently a U(1) symmetric approach has also been developed. It is a conserving gapless formalism [28].

To study numerically the trapped Bose gas within the $\mathrm{BH}$ approach, one may solve first the NLSE (2.5) or (2.19) for $\psi_{0}$ and then proceed to solve the Bogoliubov-de Gennes equations (2.8) or (2.20) for $U_{n}$ and $V_{n}$. For non-zero temperatures, self-consistency is enforced by iteration. The details of our numerical approach are discussed in the following section.

\section{The numerical approach}

In this section we outline the details of our numerical approach to solve the equations of the BH theory. We present the details of the calculation technique for the zero temperature limit since it is in this limit that most of our results have been obtained. A generalization to non-zero temperature calculations is straightforward. Several groups have studied the zero temperature problem $[13,22,24,32]$. Our work relies on a basis expansion method that we have developed [25]. Since most of the experimental traps can be well approximated at their minima by a harmonic potential, $V_{t}(r)=\frac{1}{2} M\left(\omega_{x}^{2} x^{2}+\omega_{y}^{2} y^{2}+\omega_{z}^{2} z^{2}\right)$ [3-5], we use a harmonic oscillator basis. The basis is composed of product states of three separate one-dimensional harmonic oscillators, $\phi_{n}(r)=\phi_{n_{x}}(x) \phi_{n_{y}}(y) \phi_{n_{z}}(z)$. All frequencies (energy terms) are scaled in units of the smallest trap frequency, $\omega=\min \left(\omega_{x}, \omega_{y}, \omega_{z}\right)$, and the three coordinates $(x, y, z)$ are scaled to their ground state sizes $\left(a_{x}, a_{y}, a_{z}\right)$, where $a_{x, y, z}=\sqrt{\hbar / 2 M \omega_{x, y, z}}$. We also scale the scattering length $a_{\mathrm{sc}}$ to $a=\sqrt{\hbar / 2 M \omega}$. In these units the atom-atom interaction coupling takes the dimensionless form $N_{0} u_{0} \rightarrow 8 \pi N_{0} a_{\mathrm{sc}} \sqrt{\omega_{x} \omega_{y} \omega_{z}}$.

Given a basis, one can write the condensate wave function (for $T=0$ ) as $\psi_{0}(r)=\sum_{n} a_{n} \phi_{n}(r)$. Equations (2.5) and (2.8) can be rewritten in terms of their matrix representation

$$
\begin{aligned}
& \mathcal{L} \rightarrow \mathcal{L}_{n n^{\prime}}=\int \mathrm{d} r \phi_{n}^{*}(r) \mathcal{L} \phi_{n^{\prime}}(\boldsymbol{r})=\left(\omega_{n}^{(0)}-\mu\right) \delta_{n n^{\prime}}, \\
& N_{0} u_{0}\left|\psi_{0}(\boldsymbol{r})\right|^{2} \rightarrow D_{n n^{\prime}}=\sum_{m} \sum_{m^{\prime}} a_{m}^{*} a_{m^{\prime}} I_{n m n^{\prime} m^{\prime}}
\end{aligned}
$$


where

$$
I_{n m n^{\prime} m^{\prime}}=\int \mathrm{d} r \phi_{n}^{*}(r) \phi_{m}^{*}(r) \phi_{m^{\prime}}(r) \phi_{n^{\prime}}(r),
$$

with $\omega_{n}^{(0)} \equiv n_{x} \omega_{x}+n_{y} \omega_{y}+n_{z} \omega_{z}$.

The NLSE (2.5) can now be written as a matrix eigenproblem

$$
\left(\omega_{n}^{(0)} \delta_{n n^{\prime}}+N_{0} u_{0} D_{n n^{\prime}}\right) a_{n^{\prime}}=\mu_{n} \delta_{n n^{\prime}} a_{n^{\prime}} \text {. }
$$

The Bogoliubov-de Gennes equations (2.8) can also be expressed similarly in terms of $D_{n n^{\prime}}$ and $\mathcal{L}_{n n^{\prime}}$.

We find an approximate solution to Eq. (3.4) by taking a finite subset of the basis functions $\left(N_{\text {tot }}\right)$ and solving iteratively the above equation for $\mu_{n}$ and $a_{n}$. The proper choice of the subset is dictated by the relevant symmetries of the problem. One also has to prove that the number of states within the subset basis is sufficient to obtain a reasonable numerical solution. This is most easily done by checking the change in the "final" answers as a function of the number of basis states used. The iterative procedure is as follows. An initial guess $a_{n}^{\text {old }}$ is used to generate the matrix $D_{n n^{\prime}}$. The eigenvalues and eigenvectors of this matrix are found numerically and the eigenvector corresponding to the lowest eigenvalue, $a_{n}^{\text {eigen }}$, is used to update the input vector according to the rule

$$
a_{n}^{\text {new }}=\bar{c}\left[(1-\eta) a_{n}^{\text {eigen }}+\eta a_{n}^{\text {old }}\right] \text {, }
$$

where typically $\eta \in[0,1)$ is fixed for relatively weak interacting case, and adjusted step by step (by-section) for other values of $N_{0} u_{0}$. The coefficient $\bar{c}$ ensures proper normalization. Then the vector $a_{n}^{\text {new }}$ is used as the new input for generating the $D_{n n^{\prime}}$ and the steps are repeated. If the procedure converges to a fixed point, $a_{n}^{\text {new }}=a_{n}^{\text {old }}$, this point is a solution of Eq. (3.4). The lowest eigenvalue corresponds to the chemical potential $\mu$. The rate of convergence clearly depends on the value of $\eta$ chosen as well as the initial guess for the $a_{n}$. We have achieved convergence to a fixed point for a wide range of coupling strengths $N u_{0}$ by using an appropriate $\eta$. The initial guess $a_{n}^{(0)}$ was also varied with the parameter $N u_{0}$. For moderate values of this parameter, the noninteracting ground state was chosen as the initial guess. For larger values of $N_{0} u_{0}$ one can neglect the kinetic energy term in the NLSE $[15,18]$, often referred as the Thomas-Fermi approximation (TFA). In this limit, the condensate wave function becomes the mirror image of the trapping potential,

$$
\psi_{0}(r) \propto \sqrt{\mu-V_{t}(r)} \theta\left[\mu-V_{t}(r)\right],
$$

where $\theta(x)$ is the step function, and $\mu$ is determined from the normalization condition. The solution (3.6) was found to provide a better starting point for the algorithm for large values of $N_{0} u_{0}$.

The solution for the NLSE (3.4) is used to solve Eq. (2.8) for $U_{n}(r), V_{n}(r)$, and $\hbar \tilde{\omega}_{n}$. For calculations of experimental interest, the computational effort is bottle-necked at the evaluation of the $D_{n n^{\prime}}$ matrices. In using this approach, two issues must be addressed: (1) a practical method of ordering the basis must be found, i.e., a mapping $n_{x}, n_{y}, n_{z} \rightarrow n$; (2) an efficient method of storing and evaluating the integrals $I_{n m n^{\prime} m^{\prime}}$ must be identified.

Without loss of generality, we can assume all $\left(U_{k}, V_{k}\right)$ to be real. By introducing the sums and differences as 


$$
\begin{aligned}
& S_{k}(\boldsymbol{r})=U_{k}(\boldsymbol{r})+V_{k}(\boldsymbol{r}), \\
& D_{k}(\boldsymbol{r})=U_{k}(\boldsymbol{r})-V_{k}(\boldsymbol{r}) .
\end{aligned}
$$

Equation (2.8) may be rewritten in a decoupled form [21] as

$$
\begin{aligned}
& \left(\mathcal{L}+3 N u_{0} \psi_{0}^{2}\right)\left(\mathcal{L}+N u_{0} \psi_{0}^{2}\right) S_{k}(r)=\left(\hbar \tilde{\omega}_{k}\right)^{2} S_{k}(r), \\
& \left(\mathcal{L}+N u_{0} \psi_{0}^{2}\right)\left(\mathcal{L}+3 N u_{0} \psi_{0}^{2}\right) D_{k}(\boldsymbol{r})=\left(\hbar \tilde{\omega}_{k}\right)^{2} D_{k}(r) .
\end{aligned}
$$

The latest form will reduce the size of the numerical computation.

The above equation yields the spectrum of quasiparticle excitations as the eigenvalues of the product operator of $\mathcal{L}+3 N u_{0} \psi_{0}^{2}(r)$ and $\mathcal{L}+N u_{0} \psi_{0}^{2}(r)$. Mathematically, $S_{k}(\boldsymbol{r})$ and $D_{k}(\boldsymbol{r})$ correspond to the left- and right-eigenvectors of the non-Hermitian product operator (although individual operators $\mathcal{L}+N u_{0} \psi_{0}^{2}$ and $\mathcal{L}+3 N u_{0} \psi_{0}^{2}$ are Hermitian, they do not commute). In the TFA and working in the classical phase space $(r, p)$ with $E_{p}=p^{2} / 2 M$, we can easily find the excitation spectra as

$$
\begin{gathered}
\widetilde{\omega}(\boldsymbol{p}, \boldsymbol{r})=\sqrt{\left(E_{p}+V_{t}-\mu+N u_{0} \psi_{0}^{2}\right)\left(E_{p}+V_{t}-\mu+3 N u_{0} \psi_{0}^{2}\right)} \\
\quad \approx \begin{cases}\sqrt{E_{p}\left(E_{p}+2 \mu-2 V_{t}\right)} \propto p, & \theta\left(\mu-V_{t}\right), \\
E_{p}+V_{t}-\mu, & \theta\left(V_{t}-\mu\right),\end{cases}
\end{gathered}
$$

where $\theta\left(\mu-V_{t}\right)$ is the step function, and the region specified by $\theta\left(\mu-V_{t}\right)$ is the inner region of the condensate with phonon-like low energy collective excitations, while the second line of $\theta\left(V_{t}-\mu\right)$ corresponds to the outer region with no condensate mean-field, where the excitations in phase space are particle-like.

We can also understand the mathematical reason for introduction of the conjugate wave function $\Phi_{0}$ described by Eq. (2.13). By putting $\widetilde{\omega}_{0}=0$ in Eq. (3.8), one may see that $S_{0}(r) \propto \psi_{0}(r)$ is a solution, and in general we have $\left[\mathcal{L}+3 N u_{0} \psi_{0}^{2}(\boldsymbol{r})\right] D_{0}(\boldsymbol{r}) \propto \psi_{0}(\boldsymbol{r})$, which corresponds to $D_{0}(\boldsymbol{r}) \propto \Phi_{0}$. A general misleading of the previous works was to consider the trivial solution of $D_{0}(\boldsymbol{r})=0$ which corresponds to the Goldstone mode of $U_{0}=V_{0}=\psi_{0}(r)$.

\subsection{Ordering of the basis states}

We choose to order the basis states such that if $n \leq n^{\prime}$ then $E_{n}=\left(n_{x} \omega_{x}+\right.$ $\left.n_{y} \omega_{y}+n_{z} \omega_{z}\right) \leq E_{n^{\prime}}=\left(n_{x}^{\prime} \omega_{x}+n_{y}^{\prime} \omega_{y}+n_{z}^{\prime} \omega_{z}\right)$. Within the degeneracy manifolds of $E_{n}$ eigenvalue, the states are sorted by (1) increasing order of the largest of the three 1D indices $\left(n_{\max }=\max \left(n_{x}, n_{y}, n_{z}\right)\right)$; (2) increasing order of the second largest of the three $1 \mathrm{D}$ indices $\left(n_{\text {mid }}=\operatorname{sum}\left(n_{x}, n_{y}, n_{z}\right)-\max \left(n_{x}, n_{y}, n_{z}\right)-\right.$ $\left.\min \left(n_{x}, n_{y}, n_{z}\right)\right)$; (3) increasing in the $n_{x}$ index. This sorting indexes the basis states in ascending order of energy using a single integer $n$ index.

In numerical computations we further decompose the states into eight sectors since the parity along each of the three coordinates $(x, y, z)$ are good quantum numbers [14]. The resulting parity sectors consists of (even $x$, even $y$, even $z$ ), (odd $x$, even $y$, even $z$ ), (even $x$, odd $y$, odd $z$ ), etc. Then the numerical diagonalization may be performed in each of the sectors, provided that the mean 
field potential profiles due to $\rho_{0}(x, y, z), \rho^{\prime}(x, y, z), \Delta_{0}(x, y, z)$, and $\Delta^{\prime}(x, y, z)$ are symmetric, which is indeed the case of the ground state of the NLSE (3.4). This numerical strategy will reduce eight times the size of the problem, since the NLSE (2.5) and the Bogoliubov-de Gennes equations (2.8) may be solved independently in each of the parity sectors. The total computation effort is then determined by the summation of all the $I_{n m n^{\prime} m^{\prime}}$ terms in the $D_{n n^{\prime}}$ expression Eq. (3.2). One may easily see that to compute all $N_{\text {tot }}^{2}$ matrix elements would, at least, require $N_{\text {tot }}^{4}$ operations, since computing each of these matrix elements requires a summation over $N_{\text {tot }}^{2}$ terms (matrix diagonalization scales with a lower power of $N_{\text {tot }}$ and we focus on leading order behavior). To perform self-consistent calculations such as those required by the Popov approximation, each iterative loop demands $N_{\text {tot }}^{4}$-order calculations. Therefore this repartition of the total basis, into the 8 distinct parity sectors, has some clear advantages.

\subsection{Indexing the $I_{i j k l}$}

The evaluation of the basis coupling matrix elements $I_{n m n^{\prime} m^{\prime}}$ is also an extremely intensive numerically effort. Since in total there are $N_{\text {tot }}^{4}$ elements, we mention that the evaluation may lead to a memory management problem too. Various approaches have been developed [13, 25, 32]. The most common one is based on the fast Gaussian quadrature algorithms. Analytic formulas are not very useful since their numerical evaluation takes longer than a direct quadrature computation. We note that, using the harmonic oscillator basis, there is an efficient scheme for ordering the $I_{n m^{\prime} m^{\prime}}$ that allows for both a compact storage as well as efficient addressing and searching.

For a harmonic oscillator basis representation the matrix elements $I_{i j k l}$ are a direct product of three one-dimensional matrix elements,

$$
I_{i j k l}=I_{i_{x} j_{x} k_{x} l_{x}}^{1 \mathrm{D}} I_{i_{y} j_{y} k_{y} l_{y}}^{1 \mathrm{D}} I_{i_{x} j_{x} k_{x} l_{x}}^{1 \mathrm{D}} \text {. }
$$

Further, the symmetry properties (to be discussed later) of the one-dimensional matrix elements $I^{1 \mathrm{D}}$ are, obviously, the same and the values of the matrix elements are related to each other by a scaling constant. This allows the full matrix element to be calculated from a single set of $I^{1 \mathrm{D}}$ elements.

The stored values of $I^{1 \mathrm{D}}$ must be ordered and indexed. Because all harmonic oscillator basis functions $\phi_{h}$ are real, any permutation of the indices will refer to elements with equal values. Therefore, we assume that each element to be indexed by four integers $h_{1} \geq h_{2} \geq h_{3} \geq h_{4} \geq 0$. Written in this way, the $I_{i_{1} j_{1} k_{1} l_{1}}^{1 \mathrm{D}}$ elements may be mapped into a one-dimensional array labeled by an single integer index "ind",

$$
I_{h_{1} h_{2} h_{3} h_{4}}^{1 \mathrm{D}} \longrightarrow I_{\text {ind }}^{1 \mathrm{D}} \text {. }
$$

This mapping is most easily explained by considering a simple case of an object with two indices, $I_{a b}$ for $a \geq b \geq 0$. For a given value of $a$, there are

$$
\sum_{b=0}^{a} b=\frac{1}{2} a(a+1)
$$

elements preceding the next $a+1$ elements which correspond to the possible values of $b$. We associate with the original two indices a single number $a, b \rightarrow$ 
$\frac{1}{2} a(a+1)+b \quad(0 \leq b \leq a)$. Generalizing this procedure to higher numbers of indices is straightforward. For the case of $I_{h_{1} h_{2} h_{3} h_{4}}^{1 \mathrm{D}}$ with $h_{1} \geq h_{2} \geq h_{3} \geq h_{4} \geq 0$ one finds

$$
\begin{aligned}
& h_{1} h_{2} h_{3} h_{4} \rightarrow \text { ind }=\frac{1}{4 !} h_{1}\left(h_{1}+1\right)\left(h_{1}+2\right)\left(h_{1}+3\right)+\frac{1}{3 !} h_{2}\left(h_{2}+1\right)\left(h_{2}+2\right) \\
& +\frac{1}{2 !} h_{3}\left(h_{3}+1\right)+h_{4} .
\end{aligned}
$$

The use of this indexing procedure allows us to store economically all of the matrix elements needed for our computations.

\subsection{Evaluation of $I_{h_{1} h_{2} h_{3} h_{4}}^{1 \mathrm{D}}$}

The wave function for a 1D-harmonic oscillator, of fundamental frequency $\omega$, in the coordinate representation is given by

$$
\phi_{n}(x)=\left(2^{n} n !\right)^{-\frac{1}{2}}\left(\frac{1}{2 \pi a^{2}}\right)^{\frac{1}{4}} \exp \left(-\frac{x^{2}}{4 a^{2}}\right) H_{n}\left(\frac{x}{\sqrt{2} a}\right),
$$

where $a=\sqrt{\hbar / 2 M \omega}$ is the size of the ground state. Then we have

$$
\begin{aligned}
& I_{h_{1} h_{2} h_{3} h_{4}}^{1 \mathrm{D}}=\left(2^{h_{1}+h_{2}+h_{3}+h_{4}} h_{1} ! h_{2} ! h_{3} ! h_{4} !\right)^{-\frac{1}{2}} \frac{1}{2 \pi a^{2}} \int_{-\infty}^{\infty} \mathrm{d} x \\
& \quad \times \exp \left(-\frac{x^{2}}{a^{2}}\right) H_{h_{1}}\left(\frac{x}{\sqrt{2} a}\right) H_{h_{2}}\left(\frac{x}{\sqrt{2} a}\right) H_{h_{3}}\left(\frac{x}{\sqrt{2} a}\right) H_{h_{4}}\left(\frac{x}{\sqrt{2} a}\right) \\
& \quad=\frac{1}{a}\left[\left(2^{h_{1}+h_{2}+h_{3}+h_{4}} h_{1} ! h_{2} ! h_{3} ! h_{4} !\right)^{-\frac{1}{2}} \frac{\sqrt{2}}{2 \pi} M_{h_{1} h_{2} h_{3} h_{4}}\right]
\end{aligned}
$$

where

$$
M_{h_{1} h_{2} h_{3} h_{4}}=\int_{-\infty}^{\infty} \mathrm{d} y \exp \left(-2 y^{2}\right) H_{h_{1}}(y) H_{h_{2}}(y) H_{h_{3}}(y) H_{h_{4}}(y) .
$$

We note that the quantity inside the square bracket in the rhs of Eq. (3.15) is dimensionless and independent of the frequency of the harmonic oscillator. The $M_{h_{1} h_{2} h_{3} h_{4}}$ integrals may be easily evaluated with the help of the generating function of the Hermite polynomials, i.e.,

$$
\mathrm{e}^{-s^{2}+2 s y} \equiv \sum_{h_{1}}^{\infty} H_{h_{1}}(y) \frac{s^{h_{1}}}{h_{1} !}
$$

and so

$$
\begin{aligned}
& \sum_{h_{1}, h_{2}, h_{3}, h_{4}=0}^{\infty} M_{h_{1} h_{2} h_{3} h_{4}} \frac{s^{h_{1}}}{h_{1} !} \frac{t^{h_{2}}}{h_{2} !} \frac{\mu^{h_{3}}}{h_{3} !} \frac{\nu^{h_{4}}}{h_{4} !} \\
& =\int_{-\infty}^{\infty} \mathrm{d} y \mathrm{e}^{-2 y^{2}} \mathrm{e}^{-s^{2}+2 s y} \mathrm{e}^{-t^{2}+2 t y} \mathrm{e}^{-\mu^{2}+\mu y} \mathrm{e}^{-\nu^{2}+2 \nu y} \\
& =\sqrt{\frac{\pi}{2}} \exp \left[\frac{(s+t+\mu+\nu)^{2}}{2}\right] .
\end{aligned}
$$


Then, the $M_{h_{1} h_{2} h_{3} h_{4}}$ are found to be the coefficients of the Taylor expansion of the rhs of Eq. (3.18). Alternatively, numerical quadratures can be developed to evaluate (3.16) [32]. Also, another equivalent analytical expression exists,

$$
\begin{aligned}
& M_{h_{1} h_{2} h_{3} h_{4}} \equiv h_{1} ! h_{2} ! \sum_{t=0}^{\min \left(h_{1}, h_{2}\right)} \frac{2^{t}}{t !\left(h_{1}-t\right) !\left(h_{2}-t\right) !} \\
& \times \frac{1}{\pi} 2^{\bar{k}-\frac{1}{2}} \Gamma\left[\bar{k}-\left(h_{1}+h_{2}-2 t\right)+\frac{1}{2}\right] \Gamma\left[\bar{k}-h_{3}+\frac{1}{2}\right] \Gamma\left[\bar{k}-h_{4}+\frac{1}{2}\right],
\end{aligned}
$$

for $h_{1}+h_{2}+h_{3}+h_{4}=2 k=2 \bar{k}+2 t$, and zero otherwise (when $h_{1}+h_{2}+h_{3}+h_{4}=$ odd number) [33].

\subsection{From $I_{n m n^{\prime} m^{\prime}}^{1 \mathrm{D}}$ to $I_{\text {eee }}^{1 \mathrm{D}}$ and $I_{\mathrm{eoo}}^{1 \mathrm{D}}$}

The above approach was initially implemented for the calculation of low energy excitations within a mean-field Bogoliubov approximation [25]. At $T=0$, only a linear computation (one loop) of the Bogoliubov-de Gennes equations was needed. To study the properties of the excitation spectra at a finite temperature (e.g. with the Popov approximation), we are forced to develop new methods for more efficient evaluation of the matrix elements given in Eq. (3.2). After dividing the basis into 8 parity sectors, it became clear that there is indeed a systematic way of reducing the overall computational effort for one loop from $N_{\text {tot }}^{4}$-order to $N_{\text {tot }}^{3}$-order. We outline this approach in this subsection.

As we mentioned earlier, in a harmonic oscillator trap, quasiparticle states have well defined parities with respect to their $(x, y, z)$ coordinates. This can be used to accomplish more efficient evaluation of the matrix elements of the type $D_{n n^{\prime}}$, Eq. (3.2), i.e. of the following types of integrals:

$$
\sum_{m} \sum_{m^{\prime}} a_{m}^{*} a_{m^{\prime}} \int \mathrm{d} \boldsymbol{r} \phi_{m}^{*}(\boldsymbol{r})\left[\rho_{0}(\boldsymbol{r}), \rho^{\prime}(\boldsymbol{r}), \Delta_{0}(\boldsymbol{r}), \Delta^{\prime}(\boldsymbol{r})\right] \phi_{m^{\prime}}(\boldsymbol{r}) .
$$

At thermal equilibrium, the converged solutions for $\psi_{0}(r)$ and $\left(U_{k}(r), V_{k}(r)\right)$ will necessarily result in parity symmetric functions for $\rho_{0}(r), \rho^{\prime}(r), \Delta_{0}(r), \Delta^{\prime}(r)$. Therefore, these functions themselves can be expanded into one particular 8 parity basis sector: the (even $x$, even $y$, even $z$ ) basis set. As an example, we formally write

$$
\rho(r)=\sum_{n} b_{n} \phi_{n}(r)
$$

where $\phi_{n}$ are the complete symmetric basis set. Suppose that the expansion parameters $b_{n}$ are known, then the calculation of matrix elements as given in Eq. (3.20) will involve products of three $\phi_{n}$ functions

$$
\sum_{n} b_{n} \int \mathrm{d} \boldsymbol{r} \phi_{m}^{*}(\boldsymbol{r}) \phi_{n}(\boldsymbol{r}) \phi_{m^{\prime}}(\boldsymbol{r})
$$

which contain only three Hermite polynomials. We denote the corresponding terms as $I_{m n m^{\prime}}\left(I_{m n m^{\prime}}^{1 \mathrm{D}}\right)$ and the reduced integral involving products of the Hermite polynomial as $M_{m n m^{\prime}}$ (compare with $M_{n m n^{\prime} m^{\prime}}$ defined in (3.16)). The $M_{m n m^{\prime}}$ is given by the following identity [33]:

$$
\int_{-\infty}^{\infty} \mathrm{d} y \exp \left(-\frac{1}{\gamma} y^{2}\right) H_{h_{1}}(y) H_{h_{2}}(y) H_{h_{3}}(y)=\sqrt{\gamma}\left(-\frac{z}{2}\right)^{-k} \Gamma\left[k+\frac{1}{2}\right]
$$




$$
\times \sum_{t=0}^{\min \left(h_{1}, h_{2}\right)} \frac{\left(-h_{1}\right)_{t}\left(-h_{2}\right)_{t} z^{t}}{t !\left(\frac{1}{2}-k\right)_{t}} F\left(2 t-h_{1}-h_{2},-h_{3} ; \frac{1}{2}-k+t ; z\right),
$$

for $\gamma=2 / 3$ and $h_{1}+h_{2}+h_{3}=2 k$ (even), where $z=[2(1-\gamma)]^{-1}$, and $(m)_{t} \equiv$ $\frac{(m+t-1) !}{(m-1) !}$ are the Pochhammer symbols. For the case $h_{1}+h_{2}+h_{3}=$ odd numbers we have $M_{h_{1} h_{2} h_{3}}=0$. The above calculation (3.22) is now a simple summation of $N_{\text {tot }}$ terms (instead of a double summation of $N_{\text {tot }}^{2}$ terms as in Eq. (3.2)). Therefore, the computation of all matrix elements requires only $N_{\text {tot }}^{3}$ operations. Only two distinct possibilities exist for nonzero values of $M_{h_{1} h_{2} h_{3}}:(1)$ all indices are even; (2) one index is even and the other two are odd. We denote them as $M_{\text {eee }}$ and $M_{\text {eoo }}$ respectively, and they can be ordered into a one-dimensional array in a manner similar to that detailed in Sec. 3.2.

One question remains: how do we find the expansion coefficients $b_{n}$ and what is the associated computational effort? If one writes explicitly the form of expansion (3.21) for any generic term in $\rho_{0}(r), \rho^{\prime}(r), \Delta_{0}(r)$, or $\Delta^{\prime}(r)$, then one may see that these quantities involve terms proportional to $\psi_{0}^{2}(r)$ or $\left|U_{k}(r)\right|^{2}$ and $\left|V_{k}(r)\right|^{2}$. We assume that $\psi_{0}(r), U_{k}(r)$, and $V_{k}(r)$ are written as $\sum_{n} a_{n} \phi_{n}(r)$ (these are just the solutions for condensate or quasiparticle states in any of the 8 parity sector basis sets). Then we have

$$
\sum_{n} b_{n} \phi_{n}(r)=\sum_{m} \sum_{m^{\prime}} a_{m}^{*} a_{m^{\prime}} \phi_{m}^{*}(r) \phi_{m^{\prime}}(r)
$$

which allows the following expression for $b_{n}$ coefficients:

$$
b_{n}=\sum_{m} \sum_{m^{\prime}} a_{m}^{*} a_{m^{\prime}} I_{n m m^{\prime}}
$$

The above operation is again of the $N_{\text {tot }}^{3}$-order for all the expansion coefficients $b_{n}$, and the matrix elements $\int \mathrm{d} \boldsymbol{r} \phi_{m}^{*}(\boldsymbol{r}) \phi_{n}^{*}(\boldsymbol{r}) \phi_{m^{\prime}}(\boldsymbol{r})$ are exactly of the same type as those needed in Eq. (3.22), i.e. they can all be reduced to terms involving $M_{\text {eee }}$ and $M_{\text {eoo. }}$. Therefore, by first expanding terms such as $\rho_{0}(r), \rho^{\prime}(r), \Delta_{0}(r), \Delta^{\prime}(r)$ in the appropriate basis given their parity, the calculation for the density matrix elements of the type $D_{n n^{\prime}}$ has been reduced from the $N_{\text {tot }}^{4}$-order to the $2 N_{\text {tot }}^{3}$-order (one $N_{\text {tot }}^{3}$-order from calculating the expansion coefficients $b_{n}$, as in Eq. (3.25), and another $N_{\text {tot }}^{3}$-order from computing the matrix elements for the expanded form, as in Eq. (3.22)).

We want to emphasize that this technique, of reducing a calculation involving $I_{n m n^{\prime} m^{\prime}}$ to one involving only $I_{m n m^{\prime}}$, is also applicable when the ground state $\psi_{0}(\boldsymbol{r})$ (and thus $\rho_{0}(r), \rho^{\prime}(\boldsymbol{r}), \Delta_{0}(\boldsymbol{r}), \Delta^{\prime}(\boldsymbol{r})$ ) have different symmetries, as one might expect to occur in the case of vortex states. It even applies when there is no explicit symmetry. In such a case, one may use the complete set of basis states which includes all 8 different parity sectors.

\subsection{Construction of the angular momentum states}

The preceding subsections have outlined an approach which is efficient for finding the low energy excitations for a trapped Bose gas [25]. Although we have repartitioned the basis states into parity sectors for three coordinates $(x, y, z)$, 
no other special symmetries of a given trap have been used. In practice, most of the current magnetic traps used in BEC experiments have cylindrical symmetry. For such traps the angular momentum projection along the symmetric $z$-axis, $\widehat{L}_{z}$, is conserved. For $L_{z} \neq 0$ there is a double degeneracy according to the sign of $L_{z}$. Using the harmonic oscillator eigenfunctions as our expansion basis, the reconstruction of the $\widehat{L}_{z}$ eigenstates is a straightforward task [34], which will be discussed below.

For a cylindrical trap with $\omega_{x}=\omega_{y} \equiv \omega_{r}$ (radial trapping frequency), we can characterize the quasiparticle states according to (1) their energy $\widetilde{\omega}_{n} ;(2)$ their angular momentum projection $L_{z}$; and, (3) an integer indexing the sequence within a degenerate $\widetilde{\omega}_{n}$ and $L_{z}$ manifold. We notice that the states $\left(U_{k i}, V_{k i}\right)$ for $i=$ $1, \ldots, n_{d}$ correspond to the same energy $\widetilde{\omega}_{k}$ and so are any linear combinations of them (since the Bogoliubov-de Gennes equations, Eqs. (2.8) and (2.20), are linear). Thus, the eigenstates of $\widehat{L}_{z}$ into the $n_{d^{-}}$-degenerate manifold may be constructed as a linear combination, i.e. $U_{k L_{z}}=\sum c_{i} U_{k i}$ (and similarly for $V_{k i}$ ). The appropriate coefficients $c_{i}$ are given by the following system of equations:

$$
\sum_{i}\left\langle U_{k i^{\prime}}\left|\widehat{L}_{z}\right| U_{k i}\right\rangle c_{i}=L_{z} \sum_{i}\left(U_{k i^{\prime}}\left|U_{k i}\right\rangle c_{i}\right.
$$

The summation on the rhs is due to the non-orthogonality of the $U_{k i}$ functions. This is a generalized matrix eigenvalue problem. The matrix elements involved may be easily evaluated using a product of harmonic oscillator basis denoted by $\left|n_{x}\right\rangle\left|n_{y}\right\rangle\left|n_{z}\right\rangle$. Let us assume that

$$
\left|U_{k i}\right\rangle=\sum_{n_{x}, n_{y}, n_{z}} a_{n_{x}, n_{y}, n_{z}}^{k i}\left|n_{x}\right\rangle\left|n_{y}\right\rangle\left|n_{z}\right\rangle .
$$

Using the creation (annihilation) operators $\widehat{a}_{x}^{\dagger}, \widehat{a}_{y}^{\dagger}, \widehat{a}_{z}^{\dagger},\left(\widehat{a}_{x}, \widehat{a}_{y}, \widehat{a}_{z}\right)$ for the harmonic oscillator basis states $\left|n_{x}\right\rangle,\left|n_{y}\right\rangle,\left|n_{z}\right\rangle$ and the relations,

$$
\begin{aligned}
& \widehat{L}_{z}=\mathrm{i} \hbar\left(\widehat{a}_{x} \widehat{a}_{y}^{\dagger}-\widehat{a}_{x}^{\dagger} a_{y}\right), \quad a_{q}\left|n_{q}\right\rangle=\sqrt{n_{q}}\left|n_{q}-1\right\rangle, \quad q=x, y, z, \\
& a_{q}^{\dagger}\left|n_{q}\right\rangle=\sqrt{n_{q}+1}\left|n_{q}+1\right\rangle, \quad q=x, y, z,
\end{aligned}
$$

we find

$$
\begin{aligned}
& \left\langle U_{k i^{\prime}}\left|\widehat{L}_{z}\right| U_{k i}\right\rangle=\mathrm{i} \hbar \sum_{n_{x}^{\prime}, n_{y}^{\prime}, n_{z}^{\prime} n_{x}, n_{y}, n_{z}} a_{n_{x}^{\prime}, n_{y}^{\prime}, n_{z}}^{k i^{\prime} *} a_{n_{x}, n_{y}, n_{z}}^{k i} \\
& \left\langle n_{x}^{\prime}\left|\left\langle n_{y}^{\prime}\left|\left(\widehat{a}_{x} \widehat{a}_{y}^{\dagger}-\widehat{a}_{x}^{\dagger} \widehat{a}_{y}\right)\right| n_{x}\right\rangle\right| n_{y}\right\rangle=\mathrm{i} \hbar \sum_{n_{x}, n_{y}, n_{z}}\left[a_{n_{x}-1, n_{y}+1, n_{z}}^{k i^{\prime} *} a_{n_{x}, n_{y}, n_{z}}^{k i}\right. \\
& \left.\times \sqrt{n_{x}\left(n_{y}+1\right)}-a_{n_{x}+1, n_{y}-1, n_{z}}^{k i^{\prime} *} a_{n_{x}, n_{y}, n_{z}}^{k i} \sqrt{\left(n_{x}+1\right) n_{y}}\right]
\end{aligned}
$$

and

$$
\begin{aligned}
& \left\langle U_{k i^{\prime}} \mid U_{k i}\right\rangle=\sum_{n_{x}^{\prime}, n_{y}^{\prime}, n_{z}^{\prime} n_{x}, n_{y}, n_{z}} a_{n_{x}^{\prime}, n_{y}^{\prime}, n_{z}^{\prime}}^{k i_{x}^{\prime}} a_{n_{x}, n_{y}, n_{z}}^{k i} \delta_{n_{x} n_{x}^{\prime}} \delta_{n_{y} n_{y}^{\prime}} \delta_{n_{z} n_{z}^{\prime}} \\
& =\sum_{n_{x}, n_{y}, n_{z}} a_{n_{x}, n_{y}, n_{z}}^{k i^{\prime} *} a_{n_{x}, n_{y}, n_{z}}^{k i}
\end{aligned}
$$


Similar techniques may be used in the case of a spherically symmetric harmonic trap to find the quasiparticle states for the angular momentum operators $\widehat{L}^{2}=\widehat{L}_{x}^{2}+\widehat{L}_{y}^{2}+\widehat{L}_{z}^{2}$. In this case the eigenstates of $\widehat{L}^{2}$ and $\widehat{L}_{z}$ must be of a spherical harmonics type, $Y_{L L_{z}}$.

\section{Results and discussions}

We incorporated the above ideas into a numerical program and use it in the study of interacting trapped Bose gases within the standard mean-field BH approximation. In our ongoing efforts, we have solved the $\mathrm{BH}$ equations for the excitations of condensates in various types of traps. Some of the results are discussed here.
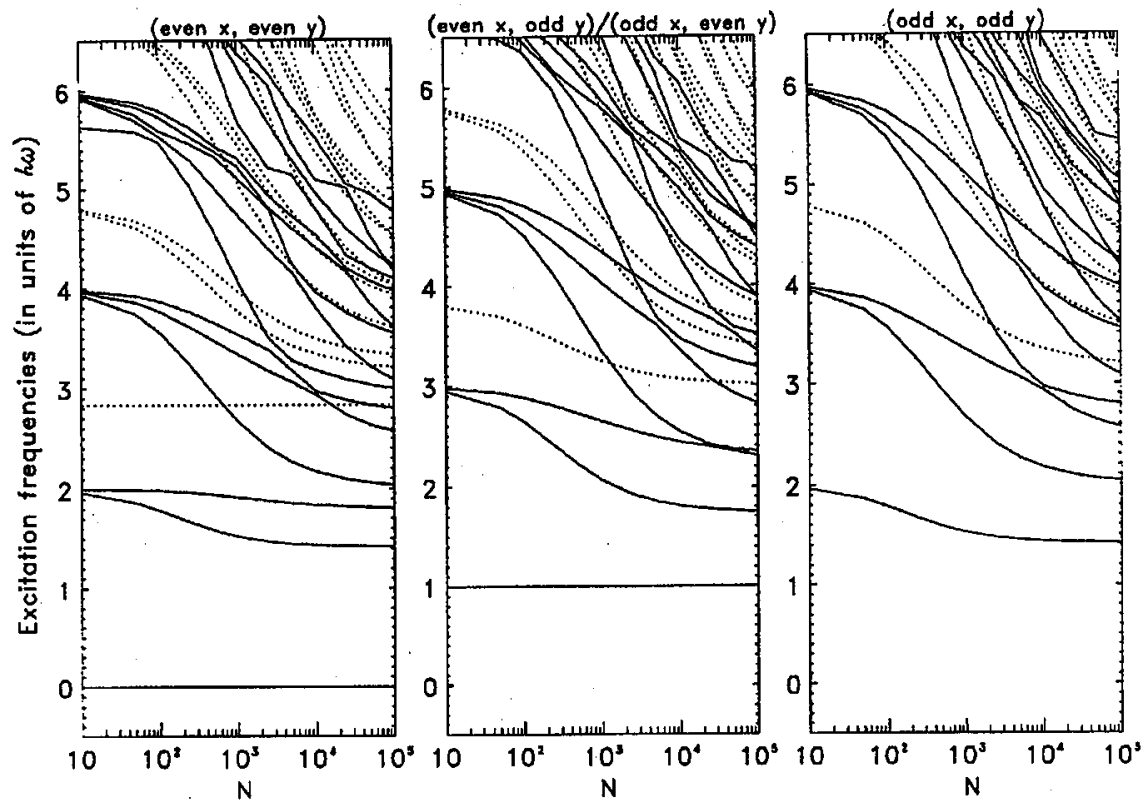

Fig. 1. The calculated dependence of quasiparticle excitation frequencies on the number of condensed atoms for the JILA TOP trap with $\left(\omega_{x}: \omega_{y}: \omega_{z}\right)=(1: 1: \sqrt{8})(129 \mathrm{~Hz})$, for ${ }^{87} \mathrm{Rb}$ atoms with $a_{\mathrm{sc}}=5.2 \mathrm{~nm}$. The three separate panels are for the 4 separate parity sectors in $x$ and $y$ coordinates respectively: first panel for (even $x$, even $y$ ); second panel for (even $x$, odd $y$ ) [same as (odd $x$, even $y$ )]; third panel for (odd $x$, odd $y$ ). The even $z$ parity states are plotted with solid lines while odd parity $z$ states are plotted with dashed lines.

We note that our quasiparticles represent elementary excitations of the quantum noise $\delta \Psi(\boldsymbol{r})\left(\delta \Psi^{\dagger}(\boldsymbol{r})\right)$, while in the current experimental investigations the quasiparticle excitations are created by using microwave pulses or trapping potential perturbations [7].

For the solution of the NLSE (2.5), we typically use up to 10,000 states with the highest energy states corresponding to $E_{\max }>50 \hbar \omega$ for the JILA TOP trap 
and $E_{\max }>200 \hbar \omega$ for the MIT cloverleaf trap. Here we only present results for the TOP trap with $\omega_{x}=\omega_{y}=\omega_{z} / \sqrt{8}$. For the zero-temperature calculations, the same number of basis states were used for Eq. (2.8), therefore, higher excitations, closer to $E_{\max }$, are not well represented. For finite temperature calculations within the Popov approximation, we have used fewer basis states for Eq. (2.20), in order to make the calculation possible on a personal workstation. We limited the calculations to a range of parameter where the convergence of the solution is ensured.

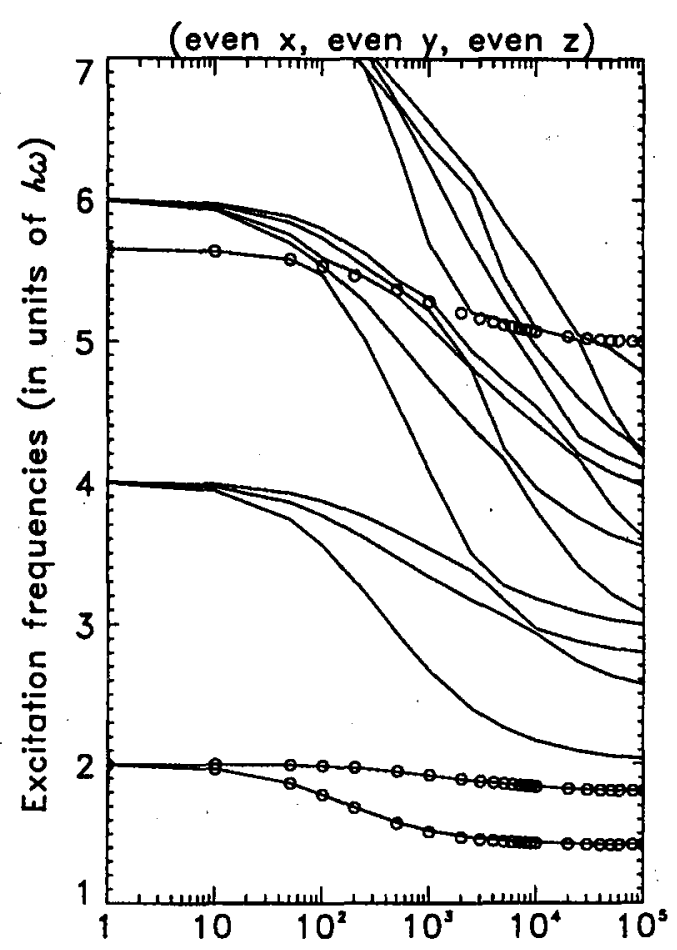

Fig. 2. The first panel of Fig. 1 for even $z$. The open circles highlight our data points for the three shape oscillation modes of Ref. [17].

The typical dependence of the excitation spectrum on the number of condensed atoms is shown in Fig. 1 calculated in the 8 separate parity sectors. The characterization of the angular momentum operator $\widehat{L}_{z}$ is a straightforward task usually involving only two energy degenerate states. All $L_{z}= \pm(2 m+1)$ states (i.e. odd angular momentum projection states) are obtained simply from the doubly degenerate states of the (odd $x$, even $y$ ) and (odd $y$, even $x$ ) sectors (of the same, $z$ parity). All states in the middle panel are doubly degenerated. This symmetry between the $x$ and $y$ basis sets requires us to solve only Eqs. (2.8) and (2.20) within the (odd $x$, even $y$ ) sector (for both odd and even $z$ parity). The even angular 

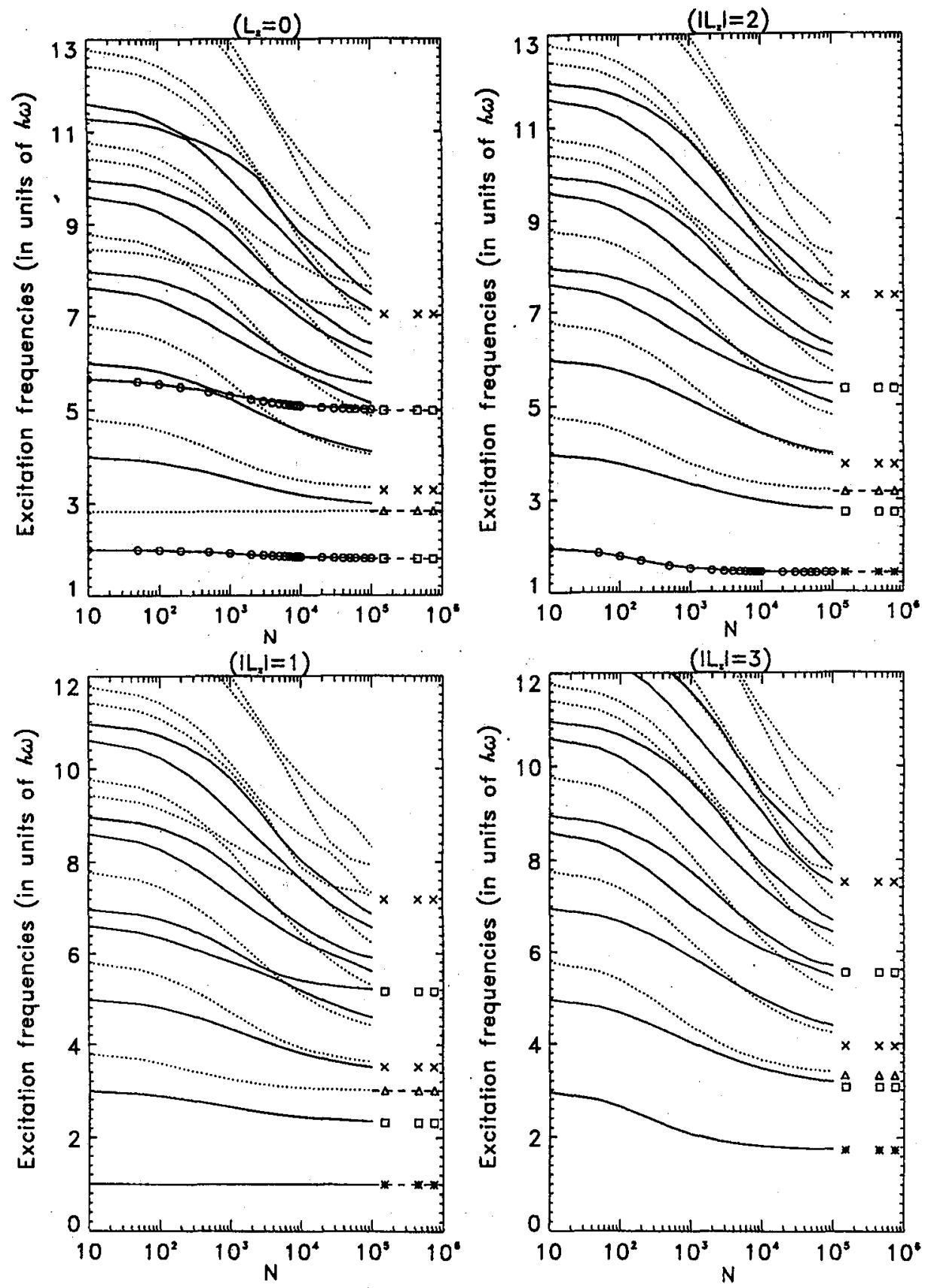

Fig. 3. The same curves as in Fig. 1, now characterized by their $L_{z}$ quantum numbers. The open circles denote the three shape oscillation modes from Ref. [17]. The dashed lines in the asymptotic limit, $N=10^{5} \div 10^{6}$, are the results based on Ref. [16]. The asterisks, triangles, squares, and X's denote, respectively, the $n=0,1,2,3$ modes in the asymptotic limit of Ref. [19]. 

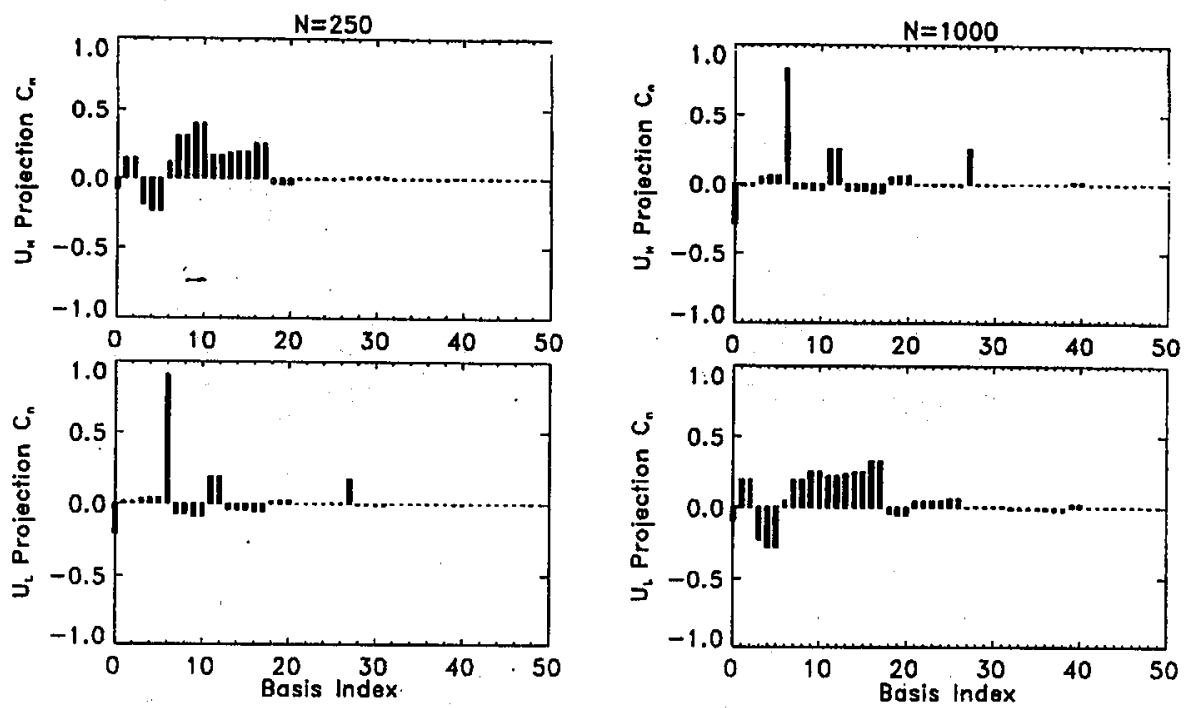

Fig. 4. Plots of the expansion coefficients of the mode function $U$ for two modes at $N=250$ and $N=1000$. These modes participate in the lowest energy possible crossing of the $L_{z}=0$ curves. The higher energy modes are shown across the top and the lower energy modes are shown across the bottom. It is interesting to note that the level crossing actually occurs at $N \approx 655$. At this point, our numerical diagonalization results in the energy difference of $\approx 0.001$.

momentum states, $L_{z}= \pm(2 m)$, are formed by the linear combinations of the solutions for (even $x$, even $y$ ) and (odd $x$, odd $y$ ) sectors (i.e. states in the first and third panel). In particular, all $L_{z}=0$ states are within the (even $x$, even $y$ ) sector. For every state in the (odd $x$, odd $y$ ) sector there is always an energy degenerate counterpart in the (even $x$, even $y$ ) sector. Together they form a nonzero even $\pm L_{z}$ pair of states. In this plot, we have connected the calculated points assuming no crossing between states with the same symmetries.

In the first panel of this Fig. 1, there are two curves with constant excitation frequencies. The lowest curve, for $\widetilde{\omega}=0$, corresponds to the Goldstone mode associated with the $U(1)$ symmetry breaking. The higher one, at $\widetilde{\omega}=\sqrt{8}$, is the small amplitude center of mass motion of the rigid cloud in the $z$-direction. Similarly, in the middle panel, the doubly degenerate state for $\tilde{\omega}=1$ corresponds to the small amplitude center of mass oscillation in the $x$ and $y$ axes.

Three of the independent shape oscillations have been measured experimentally $[7,8]$ and discussed extensively using various analytic approaches [15, 17-20]. In Fig. 2 we compare our calculations with the results of Ref. [17]. In Fig. 3 we show the dependence of the quasiparticle eigenfrequencies on $N$ for $L_{z}=0, \pm 1, \pm 2$, and \pm 3 . The open circles denote the three shape oscillation modes from Ref. [17]. The dot lines in the asymptotic limit, $N=10^{5} \div 10^{6}$, are obtained using the Stringari results [15]. The asterisks, triangles, squares, and X's denote the $n=$ $0,1,2,3$ modes, respectively, obtained in the asymptotic limit of Refs. [19, 20]. 

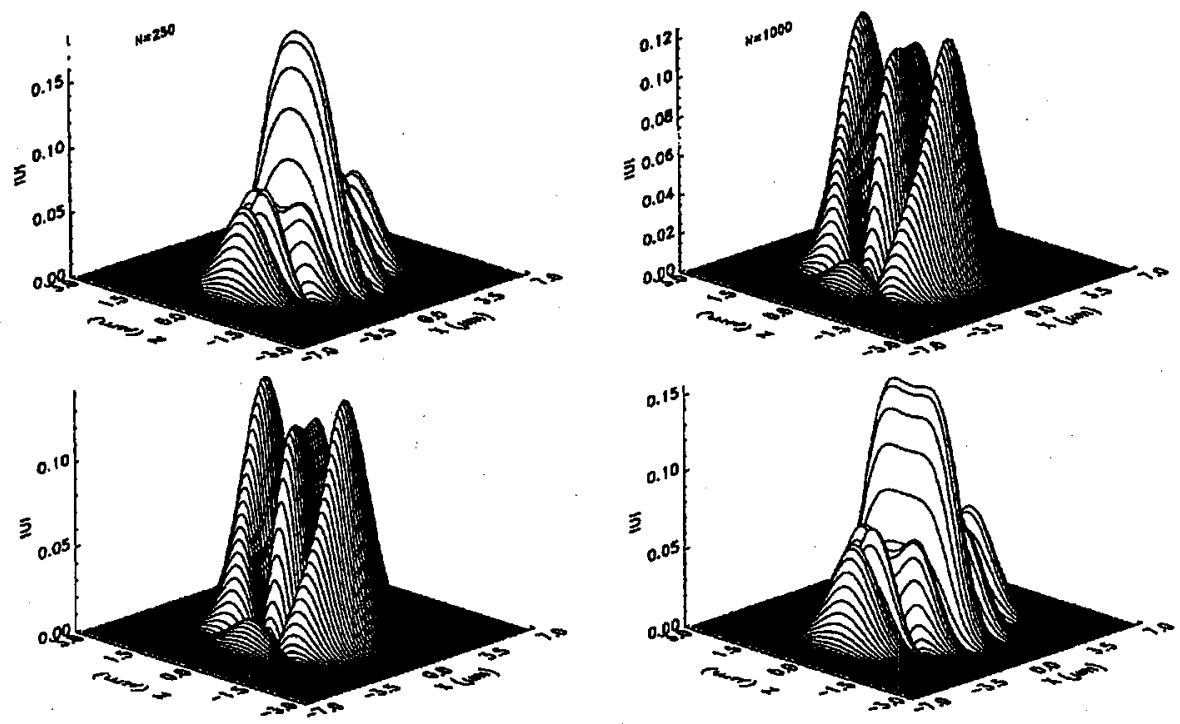

Fig. 5. The mode functions $|U(x, 0, z)|$ for the modes of Fig. 4.

Our numerical evaluation agrees well in the asymptotic limit with the analytical results of $[15,17-20]$.

In Figs. 1, 2, and 3, all crossings between curves of different $z$ parity are allowed. For curves with the same $z$ parity, we have connected the calculated points assuming that there are no crossings between them. This may not be always true. Let consider a possible crossing for the lower energy curves with $L_{z}=0$ from Fig. 3. Any of these curves represents one of the shape oscillations Ref. [17] and, therefore, they should maintain their symmetry through any value of $N$. In Fig. 4, for values of $N=250$ and $N=1000$ on either side of the possible crossing, we show projections of the mode function $U(r)$ onto the (even $x$, even $y$, even $z$ ) basis for each of the two curves involved. This may suggest that a crossing point exists in this range of value of $N$. To firmly determine if this crossing indeed exists one has to perform detailed numerical computations at exactly the crossing points (minimum distance points between the two energy curves for avoided crossings). We have performed such a study of the crossing shown in Fig. 4. Figure 5 presents the shape of $|U(z, y=0, z)|$ for the same values of $N$. These figures indicate the existence of a crossing between these two curves. In our initial studies [25] this crossing was identified as an avoided crossing based on the fact that these two quasiparticle energy levels belong to the same symmetry manifold. Recent semiclassical studies of quasiparticle motion in trapped condensates have revealed rich chaotic dynamics underlining these complicated level crossings and avoided crossings [35]. 

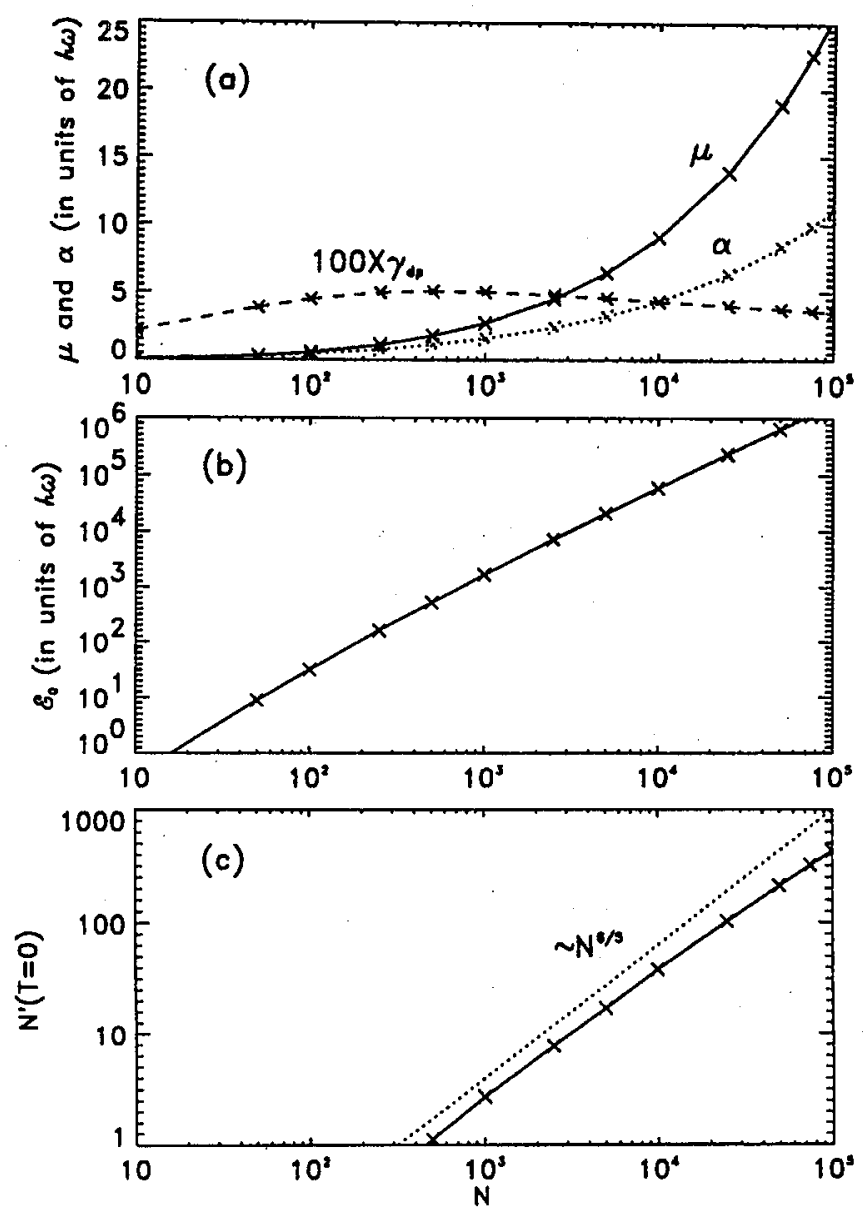

Fig. 6. Zero temperature results for (a) the chemical potential $\mu$ and the dephasing parameter $\alpha$, (b) total ground state energy, and (c) number of the noncondensate particle. The crosses denote the calculated data points.

In Fig. 6 various zero temperature results are plotted as a function of $N$. Figure 6 a presents the chemical potential, $\mu$, the dephasing parameter, $\alpha$, as well as the dephasing rate, $\gamma_{\mathrm{dp}}=\alpha / \sqrt{N_{0}}$ as a function of $N$. In the TFA both $\mu$ and $\alpha$ scale as $N^{2 / 5}$, while $\gamma_{\mathrm{dp}}$ scales as $N^{-1 / 10}$. It is interesting to note that before $\gamma_{\mathrm{dp}}$ reaches its asymptotic scaling behavior it passes through a maximum: Figure $6 \mathrm{~b}$ presents the total ground state energy as a function of $N$. Figure $6 \mathrm{c}$ presents the number of noncondensed particles in the zero temperature limit as a function of $N$. The condensate depletion is a good measure of the effect of interparticle interaction. As a percentage, only a very small fraction of the atoms are noncondensed. In the asymptotic limit $N^{\prime}(T=0)$ scales approximately as $N^{6 / 5}$, as indicated with the dashed line. This is consistent with the empirical results of Ref. [31] as well as the semiclassical results of Ref. [36]. The projection, $z_{k}$, of the condensate wave function 


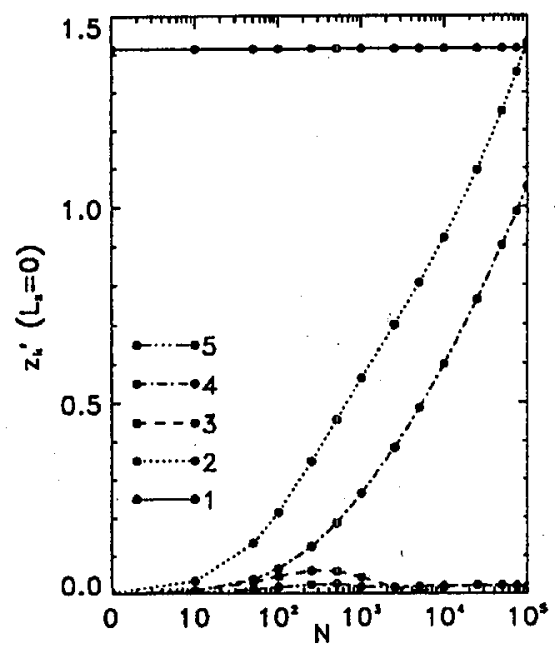

Fig. 7. The projections of the condensation wave function onto the various $L_{z}=0$ mode functions. The lists are indexed by an integer denoting in increasing order (the weakly interacting limit) the quasiparticle modes within the $L_{z}=0$ symmetry.

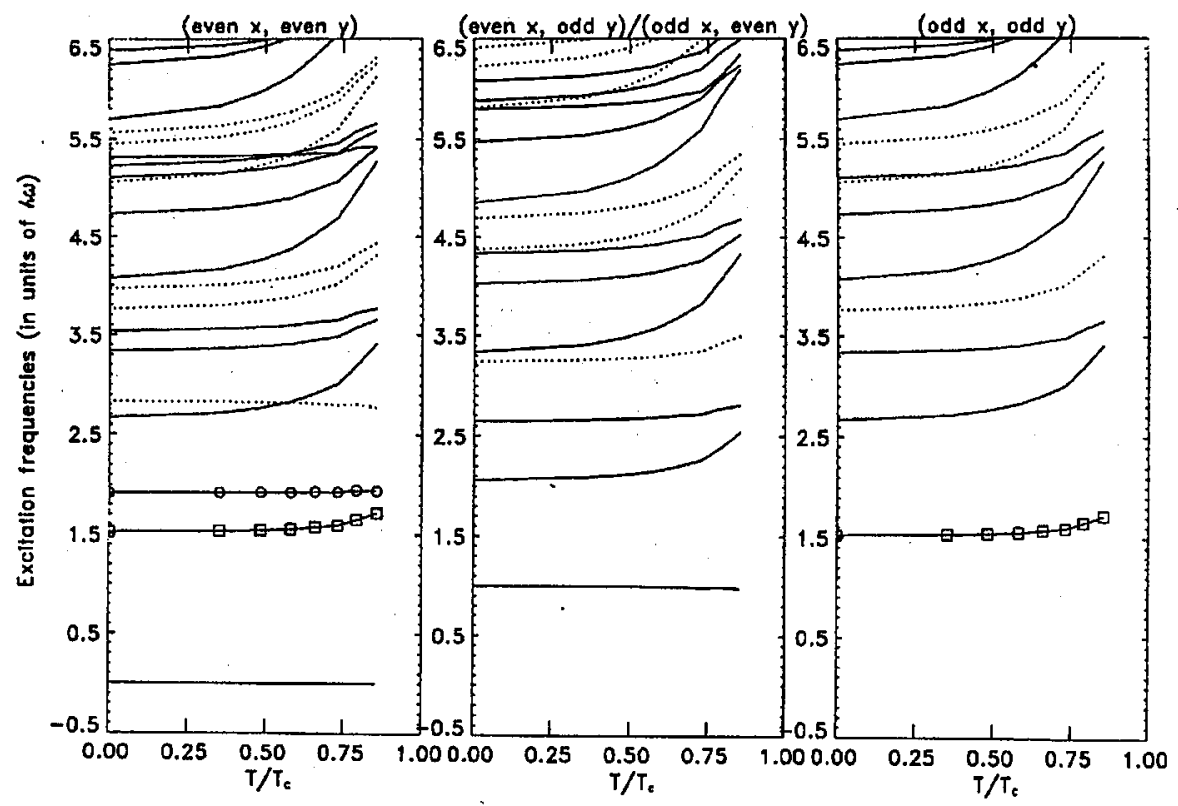

Fig. 8. The calculated dependence of quasiparticle excitation frequencies on the temperature $T$, within the Popov approximation for $N=1000$. The open circles represent the results for $L_{z}=0$ modes while the squares represent the results for $L_{z}= \pm 2$ modes. 


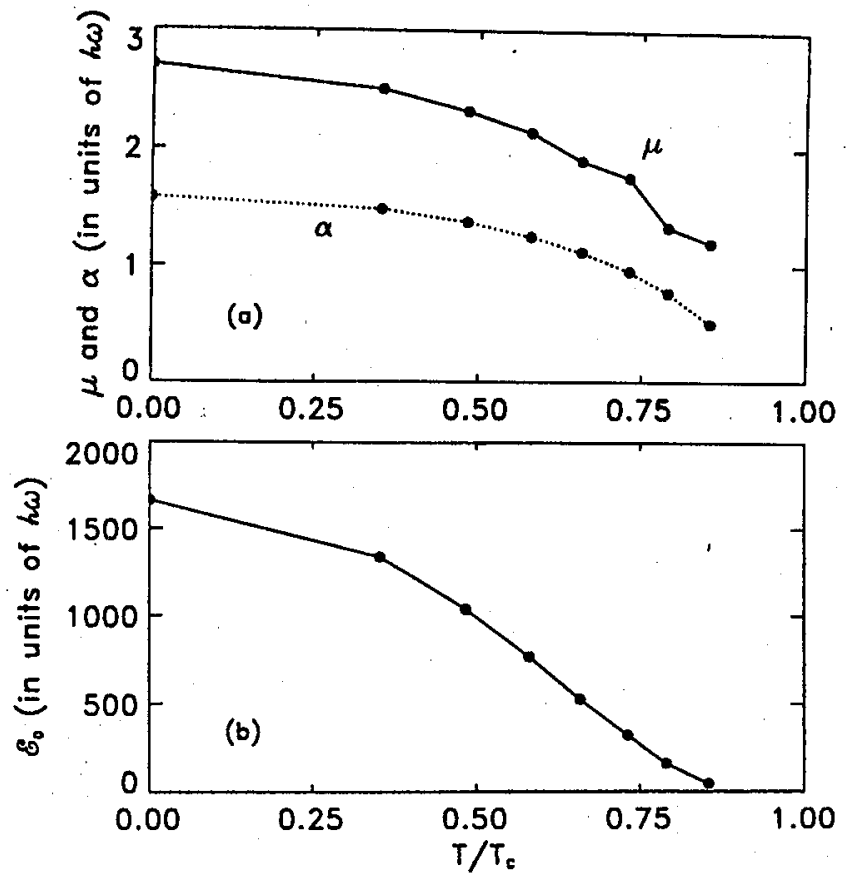

Fig. 9. The temperature dependent results for (a) the chemical potential and the dephasing, and (b) the ground state energy computed within the Popov approximation for $N=1000$ atoms. The filled circles denote the calculated data points.

onto the quasiparticle mode functions $\left(U_{k}, V_{k}\right)$ (see Eq. (2.16)), is plotted in Fig. 7 only for $k$ corresponding to the $L_{z}=0$ quasiparticles since the ground state condensate wave function $\psi_{0}(r)$ has the $L_{z}=0$ symmetry.

In Figs. 8, 9, and 10, we plot our preliminary results for finite temperature calculations within the Popov approximation for the JILA TOP trap with $N=1000$ atoms. The trap and atomic parameters are the same as in the previous zero temperature results. Figure 8 is arranged in a similar fashion as Fig. 1 except now we plot the dependence of the excitation spectra on the temperature $T$, which is determined from the self-consistent Popov calculation by specifying both $N_{0}$ and $N$. We denote with $T_{\mathrm{c}}$ the ideal Bose gas condensation temperature for the same trap parameters and number of atoms (in our case it is equal to $13.3 \hbar \omega / k$ ). Even with the limited basis set of functions used, the highest temperature point computed is very close to $T_{c}$. For $N_{0}=0.125 \mathrm{~N}$ the temperature is given approximately by $T / T_{\mathrm{c}} \propto(1-0.125)^{1 / 3} \approx 0.9565$. The open circles and squares are used to highlight the shape oscillations recently studied experimentally $[7,8]$ but they do not represent experimental data points. We can see that when $T$ is close to $T_{\mathrm{c}}$, the overall trends of the temperature dependence of the two excitation frequencies are similar to those measured in Ref. [8], although $N=1000$ atoms is significantly smaller than the total number of atoms involved in the experiment. However, close 

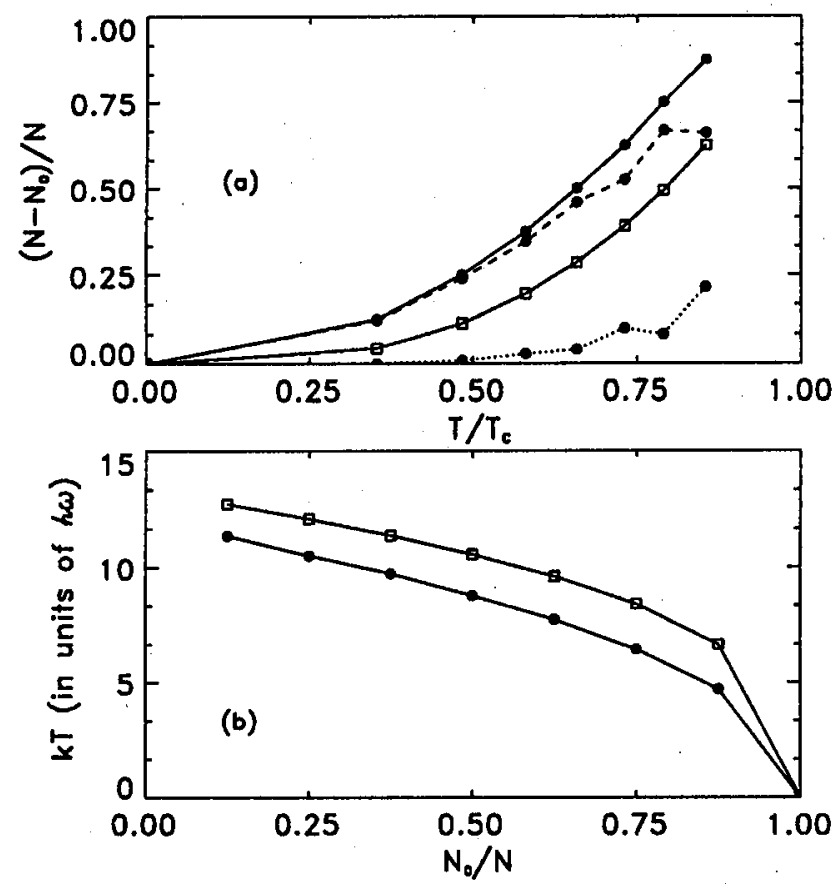

Fig. 10. (a) The depleted fraction as a function of the computed temperature within the Popov approximation. (b) The temperature as a function of the condensate fraction within the Popov approximation. Calculations were performed for $N=1000$ atoms. The filled circles denote the calculated data points and the open squares represent the results for a trapped ideal Bose gas: $N_{0} / N=1-\left(T / T_{\mathrm{c}}\right)^{3}$.

to the transition temperature, our calculations do not produce any rapid changes of the excitation frequencies. This is in disagreement with the variations observed in recent experiments and is the subject of our continued study.

Figure 9 presents: (a) the temperature dependence of the chemical potential, $\mu$, and the dephasing parameter, $\alpha$, and (b) the ground state energy of the condensate.

In Fig. 10a the solid line with filled circles shows the fraction of noncondensed atoms, $N^{\prime} / N=\left(N-N_{0}\right) / N$, plotted against the computed temperature. The filled circles denote the computed data points. The dashed line represents the fraction as given by our numerical solution using a basis of states satisfying $E_{\max } \leq 50 \hbar \omega$. The dotted line represents the difference between the two curves. We are confident that the size of the basis adopted here is suitable to describe the present system, of course, increasing the total number of atoms, the interaction strength, or the temperature would require a larger basis set. Figure $10 \mathrm{~b}$ presents the computed temperature of the condensed gas plotted against the fraction of condensate atoms. 
We note that the computed temperature is always lower than that of the ideal Bose gas prediction, $N_{0} / N=1-\left(T / T_{\mathrm{c}}\right)^{3}$. This is consistent with the observation of lowering the condensation temperature $T_{\mathrm{c}}$ from Ref. [39].

\section{Conclusions}

We conclude the following:

(1) At zero temperature, results of the quasiparticle excitation spectra from the mean field Bogoliubov approximation calculations agree well with the experimental data. Many approximate analytical approaches $[15,17-20]$ also produce excellent predictions for various quasiparticle excitation frequencies in the Thomas-Fermi limit.

(2) For finite temperatures, we have presented a self-consistent mean field Bogoliubov-Hartree calculation within the Popov approximation. Our preliminary calculations indicate that more detailed studies are needed in order to explain the recently measured finite temperature excitation results [8].

\section{Acknowledgments}

We would like to acknowledge many enlightening conversations with Drs. Y. Castin, M. Edwards, B. Forrey and T.A.B. Kennedy. This work is supported by the U.S. Office of Naval Research grant No. 14-97-1-0633. L.Y. also wants to acknowledge the support of NSF grant INT. 9722745. The work of W.H. is supported by the National Science Foundation through a grant for the Institute for Theoretical Atomic and Molecular Physics at Harvard University and Smithsonian Astrophysical Observatory.

\section{References}

[1] See the special issue Laser Cooling and Trapping of Atoms, J. Opt. Soc. Am. B 6 (1989), Eds. S. Chu, C.E. Wieman; and the review articles in the section of "Advances in Laser Cooling and Trapping of Atoms; Atom Optics", Proc. 14th Int. Conf. on Atomic Physics, Eds. D.J. Wineland, C.E. Wieman, S.J. Smith, AIP Press, New York 1995.

[2] J.M. Doyle, J.C. Sandberg, I.A. Yu, C.L. Cesar, D. Kleppner, T.J. Greytak, Phys. Rev. Lett. 67, 603 (1991); O.J. Luiten, M.W. Reynolds, J.T.M. Walraven, Phys. Rev. A 53, 381 (1996); W. Ketterle, N.J. Van Druten, Adv. Atom. Mol,, Opt. Phys. 37, 181 (1996).

[3] M.H. Anderson, J.R. Ensher, M.R. Matthews, C.E. Wieman, E.A. Cornell, Science 269, 198 (1995).

[4] C.C. Bradley, C.A. Sackett, J.J. Tollett, R.G. Hulet, Phys. Rev. Lett. 75, 1687 (1995).

[5] K.B. Davis, M.-O. Mewes, M.R. Andrews, N.J. van Druten, D.S. Durfee, D.M. Kurn, W. Ketterle, Phys. Rev. Lett. 75, 3969 (1995).

[6] M.R. Andrews, M.-O. Mewes, N.J. van Druten, D.S. Durfee, D.M. Kurn, W. Ketterle, Science 273, 84 (1996).

[7] D.S. Jin, J.R. Ensher, M.R. Matthews, C.E. Wieman, E.A. Cornell, Phys. Rev. Lett. 77, 420 (1996); M.-O. Mewes, M.R. Andrews, N.J. van Druten, D.M. Kurn, D.S. Durfee, C.G. Townsend, W. Ketterle, ibid. 77, 988 (1996). 
[8] D.S. Jin, M.R. Matthews, J.R. Ensher, C.E. Wieman, E.A. Cornell, Phys. Rev. Lett. 78, 764 (1997).

[9] M.R. Andrews, C.G. Townsend, H.-J. Miesner, D.S. Durfee, D.M. Kurn, W. Ketterle, Science 275, 637 (1997).

[10] E. Burt, R.W. Ghrist, C.J. Myatt. M. Holland, E.A. Cornell, C.E. Wieman, Phys. Rev. Lett. 79, 337 (1997).

[11] M.-O. Mewes, M.R. Andrews, D.M. Kurn, D.S. Durfee, C.G. Townsend, W. Ketterle, Phys. Rev. Lett. 78, 582 (1997).

[12] M.R. Andrews, D.M. Kurn, H.-J. Miesner, D.S. Durfee, C.G. Townsend, S. Inouye, W. Ketterle, Phys. Rev. Lett. 79, 553 (1997).

[13] M. Edwards, P.A. Ruprecht, K. Burnett, R.J. Dodd, C.W. Clark, Phys. Rev. Lett. 77, 1671 (1996).

[14] K.G. Singh, D.S. Rokhsar, Phys. Rev. Lett. 77, 1667 (1996).

[15] S. Stringari, Phys. Rev. Lett. 77, 2360 (1996).

[16] A.L. Fetter, Phys. Rev. A 53, 4245 (1996); Czech. J. Phys., accepted for publication.

[17] V.M. Pérez-García, H. Michinel, J.I. Cirac, M. Lewenstein, P. Zoller, Phys. Rev. Lett. 77, 5230 (1996).

[18] Yu. Kagan, E.L. Surkov, G.V. Shlyapnikov, Phys. Rev. A 54, R1753 (1996); Y. Castin, R. Dum, Phys. Rev. Lett. 77, 5315 (1996); A. Griffin, W.C. Wu, S. Stringari, Phys. Rev. Lett. 78, 1838 (1997).

[19] P. Öhberg, E.L. Surkov, I. Tittonen, S. Stenholm, M. Wilkens, G.V. Shlyapnikov, submitted to Phys. Rev. A, 1997.

[20] A.L. Fetter, D. Rokhsar, Phys. Rev. A, in press.

[21] M. Marinescu, A.F. Starace, Phys. Rev. A 56, 570 (1997).

[22] B.D. Esry, Phys. Rev. A 55, 1147 (1997).

[23] B.D. Esry, C. Green, private communication.

[24] D.A.W. Hutchinson, E. Zaremba, A. Griffin, Phys. Rev. Lett. 78, 1842 (1997); M. Edwards, private communication.

[25] L. You, W. Hoston, M. Lewenstein, Phys. Rev. A 55, R1581 (1997).

[26] A.L. Fetter, J.D. Walecka, Quantum Theory of Many-Particle Systems, McGraw-Hill, New York 1971; A.L. Fetter, Ann. Phys. (NY) 70, 67 (1972); A. Griffin, Phys. Rev. B 53, 9341 (1996).

[27] M. Lewenstein, L. You, Phys. Rev. Lett. 77, 3489 (1996); A. Imamoğlu, M. Lewenstein, L. You, Phys. Rev. Lett. 78, 2511 (1997); P. Villain, M. Lewenstein, R. Dum, Y. Castin, L. You, A. Imamoğlu, T.A.B. Kennedy, J. Mil. Opt., in press.

[28] Y. Castin, R. Dum, Phys. Rev. Lett. 79, 3553 (1997); C.W. Gardiner, Phys. Rev. A 56, 1414 (1997).

[29] J.-P. Blaizot, G. Ripka, Quantum Theory of Finite Systems, MIT Press, Cambridge MA 1986.

[30] M. Holland, D.S. Jin, M.L. Chiofalo, J. Cooper, unpublished.

[31] J. Javanainen, Phys. Rev. A 54, 3722 (1996).

[32] R.J. Dodd, J. Res. Natl. Inst. Stand. Technol. 101, 545 (1996); M. Edwards, R.J. Dodd, C.W. Clark, K. Burnett, ibid. 101, 553 (1996).

[33] I.W. Busbridge, J. London Math. Soc. 23, 135 (1948). 
[34] C. Cohen-Tannoudji, B. Diu, L. Laloë, Quantum Mechanics, Vols. 1 and 2, Wiley, New York 1997.

[35] M. Fliesser, A. Csordás, R. Graham, P. Szépfalusy, preprint, cond-mat/9707122; M. Fliesser, A. Csordás, P. Szépfalusy, R. Graham, preprint, cond-mat/9706002.

[36] F. Dalfovo, S. Giorgini, M. Guilleumas, L. Pitaevskii, S. Stringari, cond-mat/9705240, preprint.

[37] J.R. Ensher, D.S. Jin, M.R. Matthews, C.E. Wieman, E.A. Cornell, Phys. Rev. Lett. 77, 4984 (1996). 